KfK 3386 B Juli 1982

\title{
Atomic and Nuclear Properties of Materials
}

\author{
J. Engler
} Institut für Kern- und Teilchenphysik 

KERNFORSCHUNGSZENTRUM KARLSRUHE

Institut für Kern- und Teilchenphysik

KfK 3386B

Atomic and Nuclear Properties of Materials

J. Engler

Kernforschungszentrum Karlsruhe GmbH, Karlsruhe 
Als Manuskript vervielfältigt

Für diesen Bericht behalten wir uns alle Rechte vor

Kernforschungszentrum Karlsruhe GmbH

ISSN 0303-4003 
ATOMIC AND NUCLEAR PROPERTIES OF MATERIALS

\section{Abstract}

Additional comments and background material are given for the table of "atomic and nuclear properties of materials" which is published by the Particle Data Group in their "Review of Particle Properties" (Rosenfeld Tables).

ATOM- UND KERNEIGENSCHAFTEN VON MATERIALIEN

\section{Zusammenfassung}

Es werden zusätzliche Kommentare und Hintergrundmaterial fur die Tabelle "Atomic and nuclear properties of materials" zusammengestellt, die von der Particle Data Gruppe in ihrer übersicht von Teilcheneigenschaften (Rosenfeld-Tabellen) veröffentlich wird. 
In this note we give additional comments and background material to the table of "Atomic and Nuclear Properties of Materials" in the "Review of Particle Properties", as published by the Particle Data Group. The final compilation is shown in Table 1 and the following sections refer to the vertical columns of this table.

\section{Nuclear Total Cross Section $\sigma_{T}$}

The $\sigma_{T}$ are mean values of neutron nuclei cross-section in the energy range 80-240 GeV. Experimentally well-established points are taken from Murthy et al ${ }^{(1)}$ for $\mathrm{H}_{2}, \mathrm{D}_{2}, \mathrm{Be}, \mathrm{C}, \mathrm{Al}, \mathrm{Fe}, \mathrm{Cu}, \mathrm{W}$, $\mathrm{Pb}$ and $\mathrm{U}$. To find cross-sections for a tomic numbers that have not been measured two fits for interpolation are used:

a) for elements heavier than Be the authors of Ref. 1 give $\sigma_{T}=49.7 \times A^{0.77}$

b) for all nuclei a parametrization is used, which is derived from a simple optical model assuming that the absorption is proportional to the length of nuclear matter transversed. One finds $(2)$

$$
\sigma_{T}=2 \pi R^{2}\left\{1-1 / 2 k^{2}\left|1-(2 k+1) e^{-2 k}\right|\right\} \text { with } k=R / x \text {. }
$$

$R=r_{0} A^{1 / 3}$ is the nuclear radius and $x$ is the absorption length for neutrons in nuclear matter. A fit to the measured mean cross-sections gives $r_{0}=1.20 \mathrm{fm}$ and $x=2.78 \mathrm{fm}$. With these parameters the interpolation for the table was carried out, the values for all elements used are given in Table 2, column 4 . The original values taken from the measurements are underlined. A graphic presentation of the fit together with the measured cross-section is shown in Fig. 1 (upper curve). The fit agrees with the measured values within $\pm 1.5 \%$. 
A more precise interpolation is obtained when fitting the total cross-sections for each energy separately. The nuclear radius $r_{0}$ turns out to have the nearly energy independent value of $1.2 \mathrm{fm}$, which agrees satisfactorily with the value obtained from electron scattering. The nuclear absorption $x$ has a clear energy dependence as shown in Fig. 2 , which has to be taken into account if an energy dependent interpolation is needed.

2. Nuclear Inelastic Cross Section $\sigma_{I}$

The $\sigma_{I}$ are mean values of neutron nuclei cross-sections in the energy range 60-375 GeV taken from Roberts et al ${ }^{(3)}$. The inelastic cross-section is obtained from the total cross-section by subtracting a) the coherent elastic scattering $\sigma_{E}$ and $b$ ) the quasielastic scattering $\sigma_{Q}$, i.e. scattering wherein the nucleus is disrupted but there are no meson produced:

$\sigma_{I}=\sigma_{\text {Tot }}-\sigma_{I}-\sigma_{Q}$

This inelastic cross-section is of practical interest in many applications as attenuation length in matter, nuclear absorption, punch-through calculation, etc. Like for the total cross-section interpolation was done with an exponential $\sigma_{I}=39.64 \times A^{0.715}$ as given by the authors of Ref. 3 for elements heavier than Be and by the optical model formula. The measured and fitted values agree within $\pm 2 \%$ as shown in Fig. 1 (lower curve). The interpolated crosssections are given in column 5 of Table 2 .

Inelastic cross-sections for charged mesons $\pi^{+}, \pi^{-}, k^{+}, k^{-}$and for protons and antiprotons can be found in Carroll et al ${ }^{(4)}$.

3. Energy Loss for Minimum-Ionizing Particles

A) $\Delta E$ is the stopping power or the mean energy loss per path length of $1 \mathrm{~g} / \mathrm{cm}^{2}$ for particles heavier than electrons. The numbers are taken from Ref. 5 and plotted in Fig. 3. Values other than 
calculated are found by interpolation following the two curves for gases (full line) and solids (dashed line). The two curves differ as the result of the density correction term. The values for $\Delta E$ are given in column 6 of Table 2, the calculated values are underlined. For compounds and mixtures with an atomic number $A_{1+2}$ the values are calculated according to

$$
\left.\Delta E\left(A_{1+2}\right)=\left(\Delta E\left(A_{1}\right) \times A_{1}+\Delta E\left(A_{2}\right) \times A_{2}\right)\right) / A_{1+2}
$$

This relation holds within a few percent (Bragg rule) - see Ref. 6. The values for various compounds are given in Table 3.

B) Ep is the most-probable energy deposited on a path length of $1 \mathrm{~cm}$. For calculation Landau's formula with the modification of Maccabee and Papworth $(7)$ is used

$$
\begin{aligned}
& E p=\frac{A x}{\beta^{2}}\left|\ln \left(\frac{2 m c^{2}}{I^{2}} \times \frac{(B \gamma)^{2}}{\beta^{2}} \times A x\right)+0.198-\beta^{2}-\delta\right| \\
& A=0.153 \times Z / A \times \rho \text { with } \rho=\text { density in } \mathrm{g} / \mathrm{cm}^{3}
\end{aligned}
$$

For minimum ionization $\beta=0.96$ and $\gamma=3.4$ are taken, the density correction has been neglected (it would amount to approximately $3 \%$ for 1 ight and $1 \%$ for heavy nuclei).

The calculated values for Ep together with the mean excitation energy I used are given in Table 2. The values of I follow the expression $\mathrm{I} / \mathrm{Z}=9.76+58.8 \mathrm{Z}^{-1.19}$ as given by Sternheimer ${ }^{(8)}$. A more recent best guess can be found in Ref. 9 .

For compounds the mean excitation energy is given as

$$
\ln I=\sum_{k} f_{k} I_{k}
$$

where $f_{k}$ is the fractional number of electrons in the $k^{\text {th }}$ atomic species with excitation potential $I_{k^{\prime}}$ The mean value of $Z / A$ is 
given by

$$
Z / A=\sum_{k} n_{k}(Z / A)_{k},
$$

where $n_{k}$ is the partial density of the $k^{\text {th }}$ atomic component. Experimental results for Ep agree within the experimental uncertainties with the values calculated by the formula - see, e.g., for NaI the measurements of Bellamy et al ${ }^{(10)}$ and for gases the article of Cobb et al ${ }^{(11)}$.

The values for compounds are given in Table 3.

\section{Radiation Length}

The values given in the tables are from the most recent calculation of Y.S. Tsai (12). The figures refer to the infinite energy limit. A correction table for finite energy can be found in Tsai's article, the corrections amount to $5-10 \%$ at $1 \mathrm{GeV}$.

For chemical compounds or mixtures of molecules with the atomic weight $A_{12}=A_{1}+A_{2}$ the formula

$$
\frac{A_{12}}{X_{12}}=\frac{A_{1}}{X_{1}}+\frac{A_{2}}{X_{2}}
$$

was used. The values of Tsai refer strictly only to free atoms; effects of molecular biridings, crystal structures, polarization of the medium, etc. are ignored. Calculations for molecules have been performed for $\mathrm{H}_{2}$ by Bernstein and Panofsky (13), who find a $2.8 \%$ decrease of the radiation length and for $\mathrm{N}_{2}$ and $\mathrm{O}_{2}$ by Genannt and Pilkuhn (14), who find a $5.3 \%$ and $4.4 \%$ decrease. For the values for $\mathrm{H}_{2}, \mathrm{D}_{2}, \mathrm{~N}_{2}, \mathrm{O}_{2}$ and air quoted in the tables these reduction factors have been applied to the values of Tsai. For a discussion of effects in crystal structures see the review in the book of Ter-Mikaelian (15). 
5. Density and Refractive Index

- Values can be found in a variety of standard works and encyclopaedias. For the compilation the following handbooks were used:

Handbook of Chemistry and Physics, 61st edition, CRC-Press 1980-81

American Institute of Physics Handbook, 3rd edition, McGraw-Hi11, 1972

Landolt-Börnstein, 6th edition, Springer Verlag, Berlin, Heidelberg, New York, 1972

D'Ans-Lax, Taschenbuch für Chemiker und Physiker, 3rd edition,

Springer Verlag, Berlin, Heidelberg, New York, 1967

Gas Encyclopaedia, Elserier, Amsterdam, 1976

- Deuteron values are given in:

Advances of Cryogenics Engineering 15 (1970) 65

- $\mathrm{H}_{2}$ and $\mathrm{H}-\mathrm{Ne}$ mixtures in

R.W. Newport, "Bubble chamber technology", Proc. Int. Conf. on Bubble Chamber Techniques, June 1970, ANL.

- Propane values in:

R.P. Shutt, "Bubble and Spark Chambers", Vol. I, pg. 167, Academic Press 1967

- Information on bubble chamber operation and physical constants of liquids by:

G. Harige 1, CERN-BEBC,

- on standard shielding by:

K. Göbel, CERN-SPS. 


\section{References}

( 1 ) P.V.R. Murthy, C.A. Ayre, H.R. Gustafson, L.W. Jones and M.J. Longo, Nuc7. Phys. B 92 (1975) 269-308

( 2) F. Mönnig and H. Schopper in Landolt-Börnstein, New Series, Springer Verlag, Berlin, Heidelberg, New York 1973, Vol. 7, pg. 12-21

(3) T.J. Roberts, H.R. Gustafson, L.W. Jones, M.J. Longo and M.R. Whalley, Nuc1. Phys. B 159 (1979) 56-66

(4) A.S. Carroll et a1., Phys. Lett. 80 B (1979) 319-322

(5) W.H. Barkas and M.J. Berger, "Tables of Energy Losses and Ranges of Heavy Charged Particles", NASA SP-3013 (1964)

(6) H. Bichse1, "Radiation Dosemetry", Attix and Roesch, editors, Academic Press Inc., New York 1968, vol. 1, chap. 4

( 7 ) H.D. Maccabee and D.G. Papworth, Phys. Lett. 30 A (1969) 241-242

(8) R.M. Sternheimer, Phys. Rev. 145 (1966) 247-50

(9) H. Bichsel in "Am. Inst. Phys. Handbook", 3rd edition 1972, pg. $8-147$

(10) E.H. Bellamy, R. Hofstadter, W. Lakin, J. Cox, M.L. Perl, W.T. Toner and T.F. Zipf, Phys. Rev. 164 (1967) 417-420

(11) J.H. Cobb, W.W.M. AlTison and J.N. Bunch, Nucl.Instr. Meth. 133 (1976) 315-323

(12) Y.S. Tsai, Rev. Mod. Phys. 46 (1974) 815 and erratum in Rev. Mod. Phys. 49 (1977) 421

(13) D. Bernstein and W.K.H. Panofsky, Phys. Rev. 102 (1962) 522

(14) R. Genannt and H. Pilkuhn, Proceedings 13th Int. Cosmic Ray Conf. (Denver 1973) pg. 2434

(15) M.L. Ter-Mikaelian, "High-Energy Electromagnetic Processes in Condensed Media", Wiley-Intersience, New York 1972 
Figures and Table Captions

Figure 1: Neutron nuclei total and inelastic cross-sections. The curves show the optical model fit as described in the text.

Figure 2: The parameter $r_{0}$ and $x$ (= attenuation length in nuclear matter) of the optical model fit. The values for 11,14 and $21 \mathrm{GeV}$ are taken from Ref. 2 .

Figure 3: The energy loss for minimum-ionizing particles heavier than electrons as taken from Ref. 5. The interpolation lines are hand drawn, the solid line connects values for gases and the dashed line connects values for Tiquids and solids.

Table 1: Final compilation

Table 2: Compilation for elements including in the lower part elements occuring only in compounds of Table 1 . The underlined cross-sections are measured values. The underlined values for $\Delta E$ and $I$ are taken from Ref. 5 .

Table 3: Compilation for compounds. The underlined values for $\Delta E$ and I are taken from Ref. 5. 


\begin{tabular}{|c|c|c|c|c|c|c|c|c|c|c|c|c|}
\hline Material & 2 & A & $\begin{array}{l}\text { Nucleara) } \\
\text { total } \\
\text { cross- } \\
\text { section } \\
{ }^{\circ}{ }_{\mathrm{T}}^{\text {[barn] }}\end{array}$ & $\begin{array}{l}\text { Nuclearb) } \\
\text { inelastic } \\
\text { cross- } \\
\text { section } \\
\text { u}_{1} \text { [barn] }\end{array}$ & $\begin{array}{l}\text { Nuclear }{ }^{c)} \\
\text { collision } \\
\text { length } \\
\lambda \mathrm{T} \\
{\left[\mathrm{g} / \mathrm{cm}^{2}\right]}\end{array}$ & $\begin{array}{l}\text { Muclear }{ }^{c)} \\
\text { interaction } \\
\text { length } \\
\lambda 1 \\
{\left[\mathrm{~g} / \mathrm{cm}^{2}\right]}\end{array}$ & $\begin{array}{l}d \mathrm{dE} / \mathrm{dx} \\
{\left[\frac{\mathrm{MeV}}{\mathrm{MeV}} 2\right]}\end{array}$ & $\begin{array}{l}\mathrm{mind} d \\
\mathrm{Ep} \\
\mathrm{MeV} / \mathrm{cm} \\
\begin{array}{l}(\mathrm{keV} / \mathrm{cm}) \\
\text { for gas }\end{array}\end{array}$ & $\begin{array}{l}\text { Radiatio } \\
L_{r} \\
{\left[\mathrm{~g} / \mathrm{cm}^{2}\right]^{2}}\end{array}$ & $\begin{array}{l}\text { on length } \\
\text { rad } \\
\text { [Cra] }\end{array}$ & $\begin{array}{l}\text { Density f) } \\
{\left[\mathrm{g} / \mathrm{cm}^{3}\right]} \\
()^{\prime} \text { is for gas } \\
{[g / 1]}\end{array}$ & 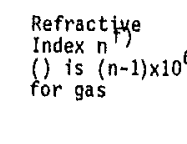 \\
\hline $\mathrm{H}_{2}$ & 1 & 1.01 & 0.0387 & 0.033 & 43.3 & 50.8 & 4.12 & $(0.19)$ & 61.28 & 865 & $0.0708(0.090)$ & $1.112(140)$ \\
\hline $\mathrm{D}_{2}$ & 1 & 2.01 & 0.073 & 0.061 & 45.7 & 53.7 & 2.07 & $(0.17)$ & 122.6 & 764 & $0.162(0.177)$ & 1.28 \\
\hline $\mathrm{He}$ & 2 & 4.00 & 0.133 & 0.102 & 49.9 & 65.1 & 1.94 & $(0.16)$ & 94.32 & 755 & $0.125(0.178)$ & $1.024(35)$ \\
\hline Li & 3 & 6.94 & 0.211 & 0.157 & 54.6 & 73.4 & 1.58 & 0.70 & 82.76 & 155 & 0.534 & - \\
\hline $\mathrm{Be}$ & 4 & 9.01 & 0.268 & 0.199 & 55.8 & 75.2 & 1.61 & 2.61 & 65.19 & 35.3 & 1,848 & - \\
\hline$c$ & 6 & 12.01 & 0.331 & 0.231 & 60.2 & 86.3 & 1.78 & 3.57 & 42.70 & 18.8 & $2.265^{9)}$ & - \\
\hline $\mathrm{H}_{2}$ & 7 & 14.01 & 0.379 & 0.265 & 61.4 & 87.8 & 1.82 & $(0.93)$ & 35.97 & 44.5 & $0.808(1.25)$ & $1.205(300)$ \\
\hline $\mathrm{O}_{2}$ & 8 & 16.00 & 0.420 & 0.292 & 63.2 & 31.0 & 1.82 & $(1.31)$ & 32.73 & 28.7 & $1.14(1.43)$ & $1.22(266)$ \\
\hline $\mathrm{Ne}$ & 10 & 20.18 & 0.507 & 0,347 & 66.1 & 96.6 & 1.73 & $(0.75)$ & 28.94 & 24.0 & $1.207(0.90)$ & $1.092(67)$ \\
\hline$A 1$ & 13 & 26.98 & 0.634 & 0.421 & 70.6 & 106.4 & 1.62 & 3.81 & 24.01 & 8.9 & 2.70 & \\
\hline Ar & 18 & 39.95 & 0.868 & 0.566 & 76.4 & 117.2 & 1.51 & 1.30 & 19.55 & 14.0 & $1.40(1.78)$ & $1.233(283)$ \\
\hline $\mathrm{Fe}$ & 26 & 55.85 & 1,120 & 0.703 & 82.8 & 131.9 & 1.48 & 10.7 & 13.84 & 1.76 & 7.87 & - \\
\hline $\mathrm{Cu}$ & 29 & 63.54 & 1.232 & 0.782 & 85.6 & 134.9 & 1.44 & 11.85 & 12.86 & 1.43 & 8.96 & - \\
\hline Sn & 50 & 118.69 & 1.967 & 1.21 & 100.2 & 163. & 1.26 & 8.3 & 8.82 & 1.21 & 7.31 & - \\
\hline Xe & 54 & 131.30 & 2.120 & 1.29 & 102.8 & 169 & 1.24 & $(3.57)$ & 8.48 & 2.88 & $3.057(5.89)$ & $(705)$ \\
\hline$W$ & 74 & 183.85 & 2.767 & 1.65 & 110.3 & 185. & 1.16 & 21.1 & 6.76 & 0.35 & 19.3 & - \\
\hline $\mathrm{Pb}$ & & 207.19 & 2.960 & 1.77 & 116.2 & 194 & 1.13 & 11.7 & 6.37 & 0.56 & 11.35 & - \\
\hline U & 92 & 238.03 & 3.378 & 1.98 & 117.0 & 199. & 1.09 & 19.3 & 6.00 & $\approx 0.32$ & $\approx 18.95$ & - \\
\hline \multicolumn{5}{|c|}{ Air $\left(20^{\circ} \mathrm{C}\right)$} & 62.0 & 90.0 & 1.82 & $(1.12)$ & 34.84 & 28993 & $\begin{array}{l}0.001205 \\
(1.29)\end{array}$ & $\begin{array}{l}1.000273 \\
(293)\end{array}$ \\
\hline \multirow{2}{*}{\multicolumn{5}{|c|}{$\begin{array}{l}\mathrm{H}_{2} \mathrm{O} \\
\text { Shielding Concrete }\end{array}$}} & 60.1 & 84.9 & 2.03 & 1.72 & 36.08 & 36.1 & 1.00 & 1.33 \\
\hline & & & & & 67.4 & 99.9 & 1.70 & 3.68 & 26.7 & 10.7 & 2.5 & - \\
\hline \multicolumn{5}{|c|}{$\mathrm{SiO}_{2}$ (quartz) } & 67.0 & 99.2 & 1.72 & 3.28 & 27.05 & 12.3 & 2.2 & 1.458 \\
\hline \multirow{4}{*}{\multicolumn{5}{|c|}{$\begin{array}{l}\mathrm{H}_{2} \text { (bubble chamber } 26^{\circ} \mathrm{K} \text { ) } \\
\mathrm{D}_{2} \text { (bubble chamber } 31^{\circ} \mathrm{K} \text { ) } \\
\mathrm{H} \text {-Ne nixture }(50 \text { nole percent) } \\
\text { Propane }\left(\mathrm{C}_{3} \mathrm{H}_{8}\right)^{\mathrm{K} \text { ) }}\end{array}$}} & 43.3 & 50.8 & 4.12 & 0.20 & 61.28 & $\approx 1000$ & $=0.063^{i)}$ & 1.112 \\
\hline & & & & & 45.7 & 53.7 & 2.07 & 0.22 & 122.6 & $\approx 900$ & $\approx 0.140^{i)}$ & 1.110 \\
\hline & & & & & 65.0 & 94.5 & 1.84 & 0.59 & 29.70 & 73.0 & 0.407 & 1.092 \\
\hline & & & & & 56.5 & 77.2 & 2.25 & $(2.54)$ & 45.38 & 111 & $0.41(2.0)$ & $1.25(1005)$ \\
\hline \multicolumn{5}{|c|}{11 ford enulsion 65} & 82.0 & 134 & 1.44 & 4.79 & 11.0 & 2.89 & 3.815 & - \\
\hline \multicolumn{5}{|l|}{$\mathrm{NaI}$} & 94.8 & 152 & 1.32 & 4.13 & 9.49 & 2.59 & 3.67 & 1.775 \\
\hline \multicolumn{5}{|l|}{ LiF } & 62 & 89.2 & 1.63 & 3.78 & 39.25 & 14.9 & 2.64 & 1.394 \\
\hline \multirow{2}{*}{\multicolumn{5}{|c|}{$\begin{array}{l}\mathrm{BGO}\left(\mathrm{Bi}_{4} \mathrm{Ge}_{3} 0_{12}\right) \\
\left.\text { Polystyrene, scintillator }(\mathrm{CH})^{\mathrm{f}}\right)\end{array}$}} & 97.4 & 156 & 1.27 & 8.07 & 7.98 & 1.12 & 7.1 & 2,15 \\
\hline & & & & & 58.4 & 82.0 & 1.95 & 1.72 & 43.8 & 42.4 & 1.032 & 1.581 \\
\hline \multirow{4}{*}{\multicolumn{5}{|c|}{$\begin{array}{l}\text { Lucite, Plexiglas }\left(\mathrm{C}_{5} \mathrm{H}_{8} \mathrm{O}_{2}\right) \\
\text { Polyethylene }\left(\mathrm{CH}_{2}\right) \\
\text { Mylar }\left(\mathrm{C}_{5} \mathrm{H}_{4} \mathrm{O}_{2}\right) \\
\text { Borosilicate glass (Pyrex })^{\mathrm{m})}\end{array}$}} & 59.2 & 83.6 & 1.95 & 1.98 & 40.55 & $\approx 34.4$ & $1.16-1.20$ & ofl . 49 \\
\hline & & & & & 56.9 & 78.8 & 2.09 & 1.68 & 44.8 & $\approx 47.9$ & $0.92-0.95$ & - \\
\hline & & & & & 60.2 & 85.7 & 1.86 & 2.24 & 39.95 & 28.7 & 1.39 & - \\
\hline & & & & & 66.2 & 97.6 & 1.72 & 3.32 & 28.3 & 12.7 & 2.23 & 1.474 \\
\hline \multicolumn{5}{|l|}{$\mathrm{CO}_{2}$} & 62.4 & 90.5 & 1.82 & $(1.92)$ & 36.2 & 20220. & 1.977 & $(410)$ \\
\hline \multicolumn{5}{|c|}{ Methane $\mathrm{CH}_{4}$} & 54.7 & 74.0 & 2,41 & $(0.91)$ & 46.5 & 64850 & $\left\{\begin{array}{l}0.423 \\
(0.717)\end{array}\right.$ & (444) \\
\hline I sobutane & $=c_{4}$ & & & & 56.3 & 77.4 & 2.22 & $(3.43)$ & 45.2 & 16930. & $(2,67)^{\prime}$ & $(1270)$ \\
\hline Freon 12 & (CC) & $\left({ }_{2} F_{2}\right)^{n)}$ & & & 70.6 & 106 & 1.62 & $(4,49)$ & 23.7 & 4810 & $(4.93)$ & $(1080)$ \\
\hline Freon 13 & $(\mathrm{CCl}$ & $\left(f_{3}\right)^{n)}$ & & & 68.1 & 101 & 1.64 & $(3.91)$ & 27.1 & 6370 & $(4.26)$ & $(720)$ \\
\hline Silica $A E$ & eroge & & & & 65.5 & 95.7 & 1.83 & 0.28 & 29.85 & sil 50. & $0.1-0.3$ & $1.0+0.25 \mathrm{\rho}$ \\
\hline
\end{tabular}

Spark or proportional chamber ${ }^{\text {P) }} \quad 0.028 \% \quad 0.020 \% \quad-0.034 \quad 0.067 \% \quad 0.019$

* Table revised Harch 82 by J, Engler. For detaj1s see Report KfK 33868, Kernforschungszentrum 075 Karisruhe, P.0.80x 3640

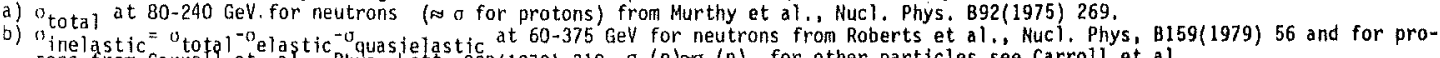

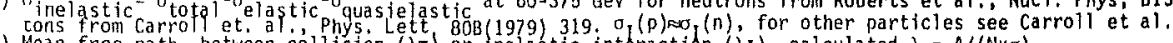

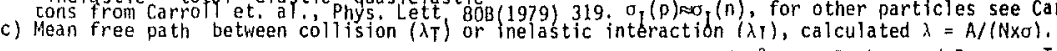

d) For minimum-ionizing protons and pions. $\Delta E$ is energy loss per $\mathrm{g} / \mathrm{cm}^{2}$ fron Barkas and Berger. Tables of Energy Losses and Ranges of Heavy Charged Particles, NASA-SP-3013(1964), for electrons see Penetration of Charged Particte

From Y.S. Tsai, Rev. Mod. Phys. 46 (1974) 815 . Corrections for molecular binding applied for $\mathrm{H}_{3}, \mathrm{D}_{2}, \mathrm{~N}$, $\mathrm{O}_{2}$ and atr.

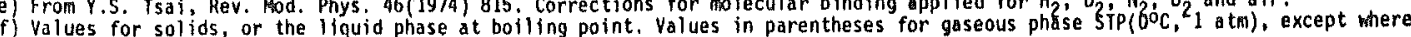

noted. Refr. active index for sodicua $D$ line.

9) For pure graphite, industrlat graphite density fay vary $2.13-2.3 \mathrm{~g} / \mathrm{cm}$. At tenuation length $\ell=115 \pm 5 \mathrm{~g} / \mathrm{cm}^{2}$, also valid for earth (typical $\rho=2,15$ ) from CERN-LRL-RHEL Shielding exp. UCRL 17841(1968),

1) Density may vary about $\mathbf{x} 3 \%$, depending on operation conditions.

j) Values for typical working condition with $\mathrm{H}_{2}$ target: 50 mole percent, $29^{\circ} \mathrm{K}, 7$ atm.

Values for typical chamber working conditions: Propane $\sim 57^{\circ} \mathrm{C}, 8-10$ atm. Freon $13 \mathrm{~B} 1 \sim 28^{\circ} \mathrm{C}, 8-10$ atm.

1) Typical scintillator; e.9. PILOT B and $\mathrm{HE} 102 \mathrm{~A}$ have an a tomfc ratio $\mathrm{H} / \mathrm{C}=1.10$.

m. Main components: $80 \% \mathrm{SiO}_{2}+12 \% \mathrm{~B}_{2} \mathrm{O}_{3}+5 \% \mathrm{Ma} \mathrm{O}^{2}$

n) Used in Cerenkov counters, values at $260 \mathrm{C}$ and $\mathrm{i}$ atm. Indices of refraction from $E . R$. Hayes, R.A. Schluter, and A. Tamosaitis,

o) $n\left(\mathrm{SiO}_{2}\right)+2 \mathrm{n}\left(\mathrm{H}_{2} \mathrm{O}\right)$ used in Cerenkov counters, $\rho=$ density in $\mathrm{g} / \mathrm{cm}^{3}$. From $\mathrm{H}$. Cantin et al., Nuc1. Instr, Meth. 118, 177(1974).

p) Values for typical construction: 2 layers $50 \mu \mathrm{m}$ Cu/Be wires, $8 \mathrm{~mm}$ gap, $60 \%$ argon, $40 \%$ isobutane or $\mathrm{CO}, 2$ layers $50 \mathrm{~mm}$ Mylar/ Aciar foils. 


\begin{tabular}{|c|c|c|c|c|c|c|c|c|}
\hline \multirow[b]{2}{*}{ Element } & \multirow[b]{2}{*}{$z$} & \multirow[b]{2}{*}{ A } & \multirow[b]{2}{*}{${ }_{\text {barn }}^{\sigma_{T}}$} & \multirow[b]{2}{*}{${ }_{\text {barn }}^{\sigma_{1}}$} & \multicolumn{2}{|c|}{$d E / d x \quad(\operatorname{Min})$} & \multirow[b]{2}{*}{$\begin{array}{l}\text { I } \\
(e V)\end{array}$} & \multirow[b]{2}{*}{$\begin{array}{l}\text { Radiation } \\
\text { Length } \\
\left(\mathrm{g} / \mathrm{cm}^{2}\right)\end{array}$} \\
\hline & & & & & $\begin{array}{l}\Delta \mathrm{E} \\
\mathrm{MeV} / \mathrm{gcm}^{-2}\end{array}$ & $\begin{array}{l}\mathrm{E}_{\mathrm{p}} \\
\mathrm{MeV} / \mathrm{cm} \\
(\mathrm{keV} / \mathrm{cm})\end{array}$ & & \\
\hline $\mathrm{H}_{2}$ & 1 & 1.01. & 0.0387 & $\underline{0.033}$ & 4.12 & $(0.190)$ & 18.7 & 61.28 \\
\hline $\mathrm{D}_{2}$ & 1 & 2.01 & $\underline{0.073}$ & 0.061 & 2.07 & $(0.165)$ & 18.7 & 122.6 \\
\hline He & 2 & 4.00 & $\underline{0.132}$ & 0.102 & 1.94 & $(0.159)$ & 42.0 & 94.32 \\
\hline Li & 3 & 6.94 & 0.211 & 0.157 & 1.58 & 0.70 & 50.3 & 82.76 \\
\hline $\mathrm{Be}$ & 4 & 9.01 & $\underline{0.268}$ & $\underline{0.199}$ & 1.61 & 2.61 & 60.0 & 65.19 \\
\hline 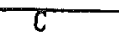 & 5 & 12.01 & 0.331 & 0.231 & 1.78 & 3.57 & 78.0 & 42.70 \\
\hline $\mathrm{N}_{2}$ & 7 & 14.01 & 0.378 & 0.265 & 1.82 & $(0.925)$ & 90.7 & 35.97 \\
\hline $0_{2}$ & 8 & 16.00 & 0.421 & 0.292 & 1.82 & $(1.314)$ & 100.9 & 32.73 \\
\hline $\mathrm{Ne}$ & 10 & 20.18 & 0.507 & 0.347 & 1.73 & $(0.747)$ & 131. & 28.94 \\
\hline Al & 13 & 26.98 & $\underline{0.634}$ & 0.421 & 1.62 & 3.81 & 163. & 24.01 \\
\hline Ar & 18 & 39.95 & 0.868 & 0.566 & 1.51 & $(1.295)$ & 210. & 19.55 \\
\hline Fe & 26 & 55.85 & 1.113 & 0.703 & 1.48 & 10.7 & 273 & 13.84 \\
\hline $\mathrm{Cu}$ & 29 & 63.54 & 1.232 & $\underline{0.782}$ & 1.44 & 11.85 & 314. & 12.86 \\
\hline Sn & 50 & 118.69 & 1.120 & 1.21 & 1.26 & 8.27 & 516. & 8.82 \\
\hline $\mathrm{Xe}$ & 54 & 131.30 & 2.120 & 1.29 & 1.24 & $(3.57)$ & 555. & 8.48 \\
\hline$W$ & 74 & 183.85 & 2.767 & $\underline{1.65}$ & 1.16 & 21.1 & 748. & 6.76 \\
\hline $\mathrm{Pb}$ & 82 & 207.19 & 2.960 & $\underline{1.77}$ & $\underline{1.13}$ & 11.7 & 826. & 6.37 \\
\hline U & 92 & 238.03 & 3.378 & 1.98 & 1.09 & 19.3 & 923. & 6.00 \\
\hline B & 5 & 10.8 & 0.305 & 0.219 & 1.67 & & 75. & 52.69 \\
\hline $\mathrm{F}$ & 9 & 19.0 & 0.484 & 0.332 & 1.65 & & 120. & 32.93 \\
\hline $\mathrm{Na}$ & 11 & 22.99 & 0.563 & 0.381 & 1.63 & & 140. & 27.74 \\
\hline Si & 14 & 28.09 & 0.660 & 0.440 & 1.67 & & 170. & 21.82 \\
\hline $\mathrm{Cl}$ & 17 & 35.45 & 0.792 & 0.520 & 1.58 & & 198. & 19.28 \\
\hline $\mathrm{Ca}$ & 20 & 40.08 & 0.871 & 0.567 & 1.63 & & 228. & 16.14 \\
\hline $\mathrm{Ge}$ & 32 & 72.6 & 1.368 & 0.861 & 1.37 & & 345. & 12.25 \\
\hline $\mathrm{Br}$ & 35 & 79.91 & 1.470 & 0.920 & 1.35 & & 374. & 11.42 \\
\hline $\mathrm{Kr}$ & 36 & 83.8 & 1.523 & 0.951 & $\underline{1.35}$ & & 381. & 11.37 \\
\hline $\mathrm{Ag}$ & 47 & 107.8 & 1.835 & 1.13 & 1.32 & & 487. & 8.97 \\
\hline$I$ & 53 & 126.9 & 2.067 & 1.26 & 1.23 & & 547. & 8.48 \\
\hline $\mathrm{Au}$ & 79 & 197.0 & 2.842 & 1.71 & 1.15 & & 797. & 6.46 \\
\hline $\mathrm{Bi}$ & 83 & 209 & 2.964 & 1.78 & 1.13 & & 835. & 6.29 \\
\hline
\end{tabular}

Table 2: Compilation for elements including in the lower part elements occuring only in compounds of Table 1. The underlined cross-sections are measured values. The underlined values for $\Delta E$ and I are taken from Ref. 5 . 


\begin{tabular}{|c|c|c|c|c|c|c|c|}
\hline \multirow[b]{2}{*}{ Ma terial } & \multirow[b]{2}{*}{$Z / A$} & \multirow[b]{2}{*}{${ }_{\mathrm{g} / \mathrm{cm}^{2}}$} & \multirow[b]{2}{*}{${ }_{\mathrm{g} / \mathrm{cm}^{2}}$} & \multicolumn{2}{|c|}{$\mathrm{dE} / \mathrm{dx} \quad(\min )$} & \multirow[b]{2}{*}{$\begin{array}{l}\text { I } \\
\text { eV }\end{array}$} & \multirow[b]{2}{*}{$\begin{array}{l}\text { Radiation Length } \\
\mathrm{g} / \mathrm{cm}^{2}\end{array}$} \\
\hline & & & & $\begin{array}{l}\Delta \mathrm{E} \\
\mathrm{MeV} / \mathrm{gcm}^{-2}\end{array}$ & $\begin{array}{c}\text { Ep } \\
\mathrm{MeV} / \mathrm{cm} \\
(\mathrm{KeV} / \mathrm{cm})\end{array}$ & & \\
\hline Air & .50 & 62.0 & 90.0 & 1.82 & $(1.12)$ & 86.8 & 34.84 \\
\hline $\mathrm{H}_{2} \mathrm{O}$ & .555 & 60.1 & 84.9 & 2.03 & 1.72 & 65.1 & 36.08 \\
\hline Concrete & 0.5 & 67.4 & 99.9 & 1.70 & 3.3 & 12.8 & 26.7 \\
\hline $\mathrm{SiO}_{2}($ quartz $)$ & .50 & 67.0 & 99.2 & 1.72 & 3.28 & 128.7 & 27.05 \\
\hline $\mathrm{H}_{2}\left(26^{\circ} \mathrm{K}\right)$ & 1.0 & 43.3 & 50.8 & 4.12 & 0.198 & 18.7 & 61.28 \\
\hline $\mathrm{D}_{2}\left(31^{0} \mathrm{~K}\right)$ & .5 & 45.7 & 53.7 & 2.07 & 0.221 & 18.7 & 122.6 \\
\hline $\mathrm{H}-\mathrm{Ne}(50 \%)$ & .524 & 65.0 & 94.5 & 1.84 & 0.588 & 110 & 29.7 \\
\hline Propane & .591 & 56.5 & 77.2 & 2.25 & $(2,54)$ & 50.3 & 45.38 \\
\hline Emulsion & .454 & 82.0 & 134. & 1.44 & 4.79 & 307. & 11.0 \\
\hline $\mathrm{NaI}$ & .427 & 94.8 & 152 . & 1.32 & 4.13 & 433. & 9.49 \\
\hline LiF & .463 & 62. & 89.2 & 1.63 & 3.78 & 96.5 & 39.25 \\
\hline$B G O$ & .420 & 97.4 & 156. & 1.27 & 8.07 & 482. & 7.98 \\
\hline Scintillator & .538 & 58.4 & 82.0 & 1.95 & 1.72 & 63.6 & 43.8 \\
\hline Lucite & .540 & 59.2 & 83.6 & 1.95 & 1.98 & 65.6 & 40.55 \\
\hline Polye thylene & .571 & 56.9 & 78.8 & 2.09 & 1.68 & $\underline{54.6}$ & 44.8 \\
\hline Mylar & .521 & 60.2 & 85.7 & 1.86 & 2.24 & 75.5 & 39.95 \\
\hline Pyrex & .50 & 66.2 & 97.6 & 1.72 & 3.32 & 128. & 28.3 \\
\hline $\mathrm{CO}_{2}$ & .50 & 62.4 & 90.5 & 1.82 & $(1.92)$ & 85.9 & 36.2 \\
\hline Methane & .625 & 54.7 & 74.0 & 2.41 & $(0.91)$ & 44.1 & 46.5 \\
\hline Isobutane & .586 & 56.3 & 77.4 & 2.22 & $(3.43)$ & 51.2 & 45.2 \\
\hline Freon 12 & .480 & 70.6 & 106. & 1.62 & $(4.49)$ & 154. & 23.7 \\
\hline Freon 13 & .479 & 68.1 & 101. & 1.64 & $(3.91)$ & 135. & 27.1 \\
\hline Silice Aeroge & .520 & 65.5 & 95.7 & 1.83 & 0.28 & 98. & 29.85 \\
\hline
\end{tabular}

Table 3: Compilation for compounds. The underlined values for $\Delta E$ and 1 are taken from Ref. 5 . 


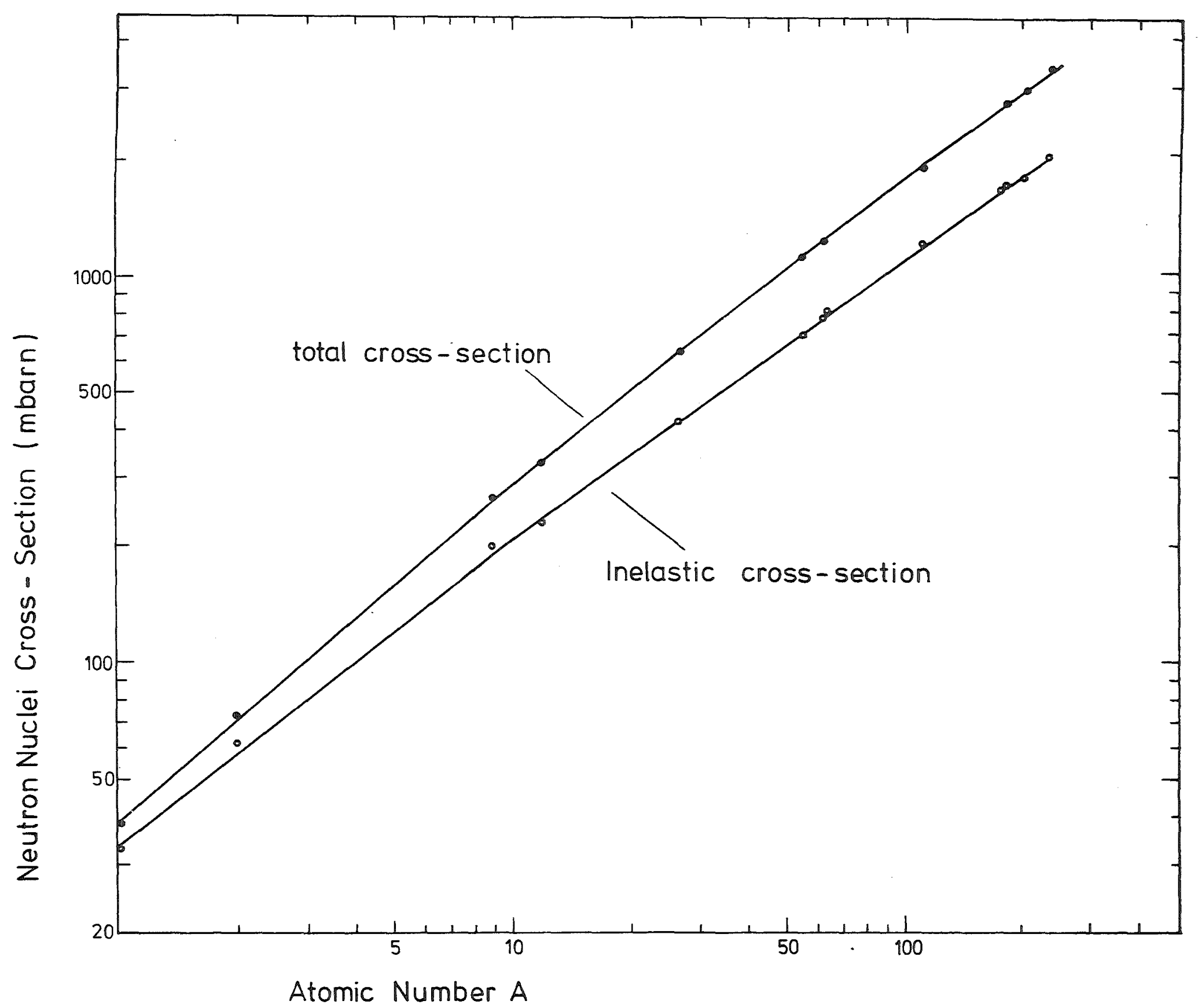

Figure 1: Neutron nuclei total and inelastic cross-sections. The curves show the optical model fit as described in the text. 


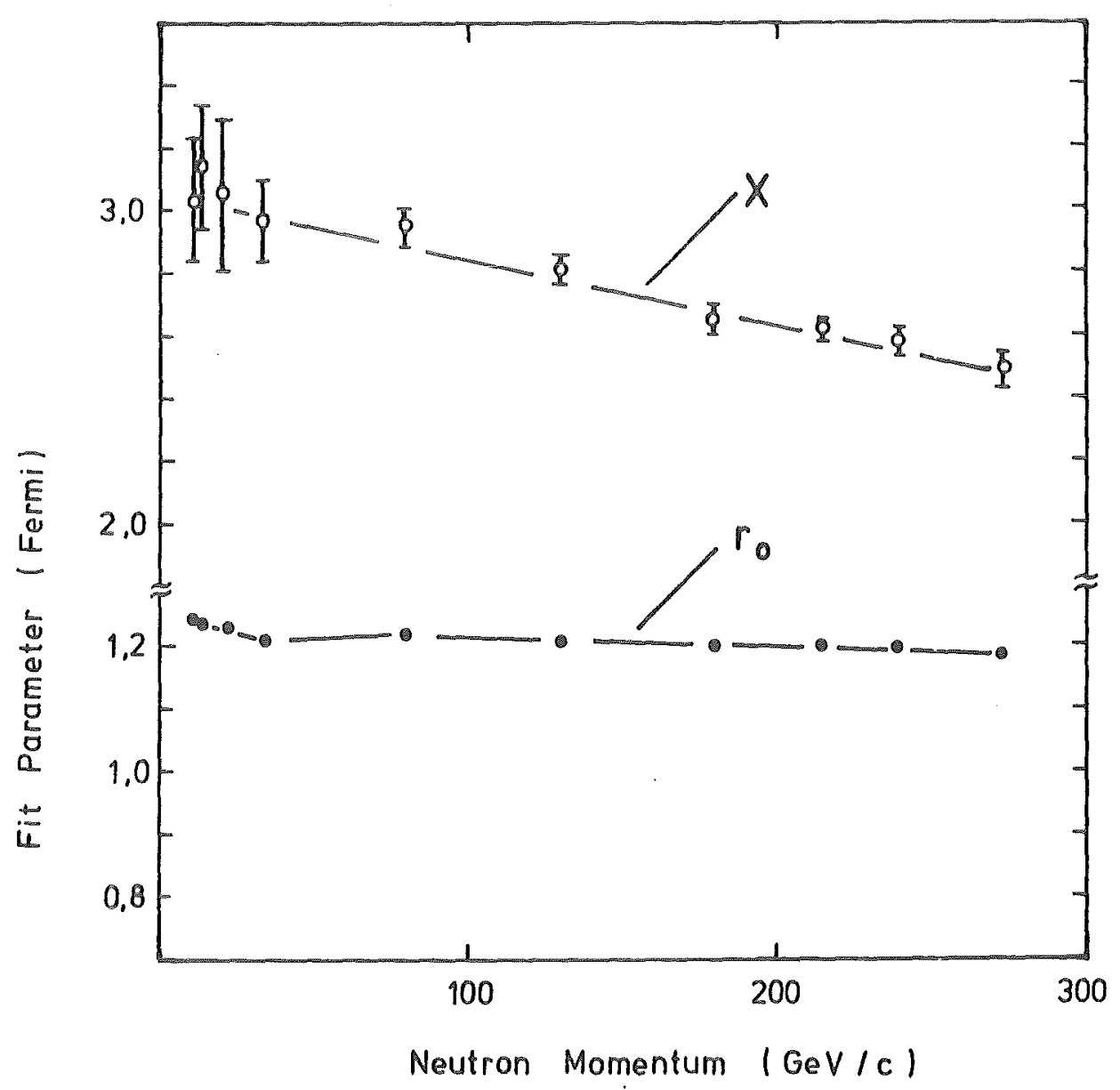

Figure 2: The parameter $r_{0}$ and $x$ (= attenuation length in nuclear matter) of the optical model fit. The values for 11,14 and $21 \mathrm{GeV}$ are taken from Ref. 2. 


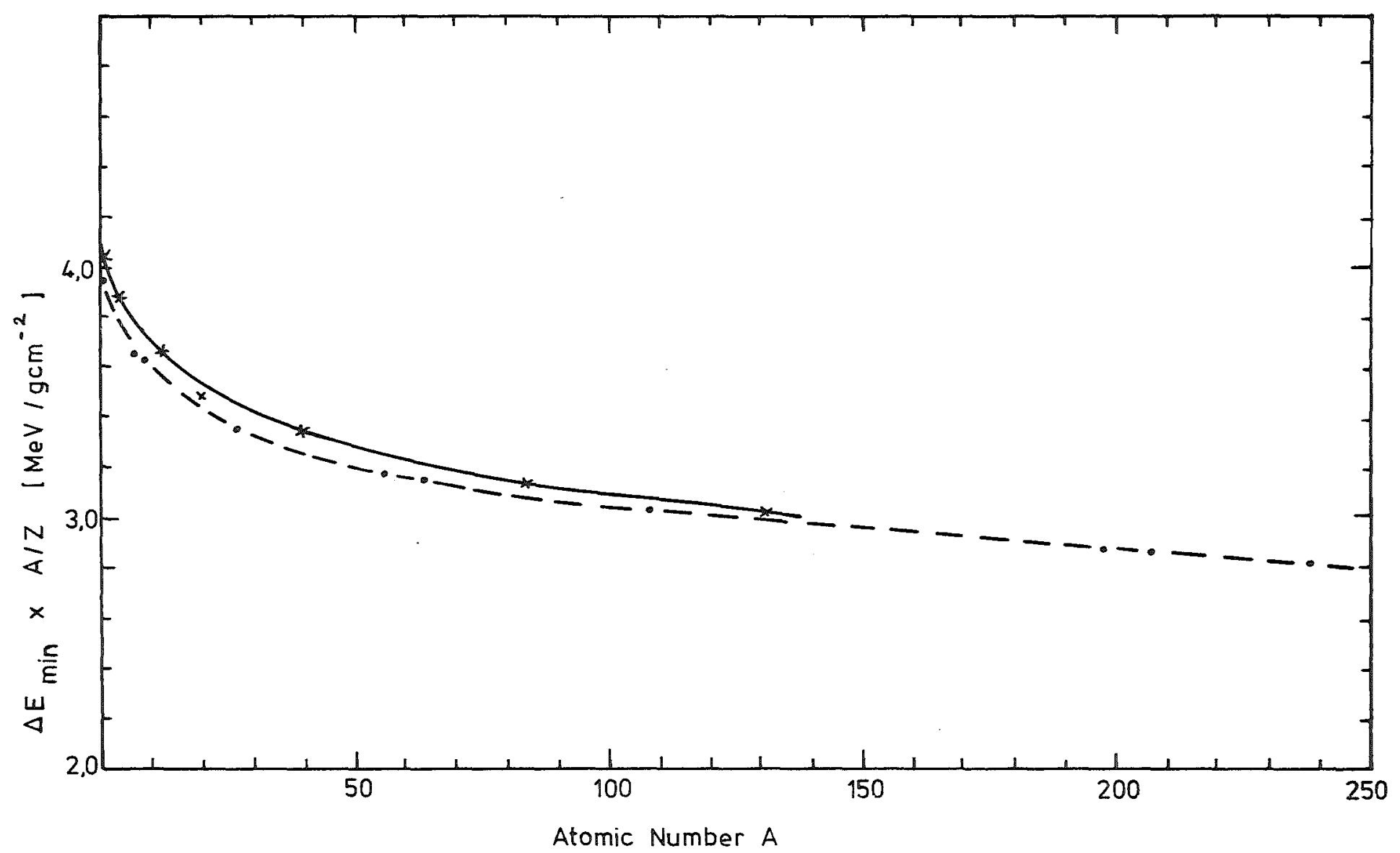

Figure 3: The energy loss for minimum-ionizing particles

heavier than electrons as taken from Ref. 5. The inter-

polation lines are hand drawn, the solid line connects

values for gases and the dashed line connects values for

liquids and solids. 